\title{
Teams merge for dark-energy mission
}

A competition between groups hoping to design a space telescope to investigate how the Universe is expanding over time has been scrapped by NASA and the US Department of Energy (DoE).

Instead, the agencies are pursuing a government-built, government-led design for the Joint Dark Energy Mission (JDEM), which may accommodate elements from all three of the teams. "It's a do-over for all of us," says Michael Levi, who is co-principal investigator for the Supernova Acceleration Probe (SNAP), a team that, he says, all of a sudden doesn't really exist any more.

NASA had been giving money to SNAP and two other groups, called the Advanced Dark Energy Physics Telescope (ADEPT) and the Dark Energy Space Telescope (Destiny). Each team was pursuing a proprietary telescope design, emphasizing different methods (see 'The telescope teams') for seeking constraints on the mysterious energy that is thought to be accelerating the expansion of the Universe. The mission is pegged for launch in 2015.

However, on 12 September, NASA and the DoE announced they will develop a common "reference design" that would not preclude any of the three methods. The design will be worked out by a new programme office opened at Goddard Space Flight Center in Greenbelt, Maryland, and a science coordination group of 12-20 people. The membership could be

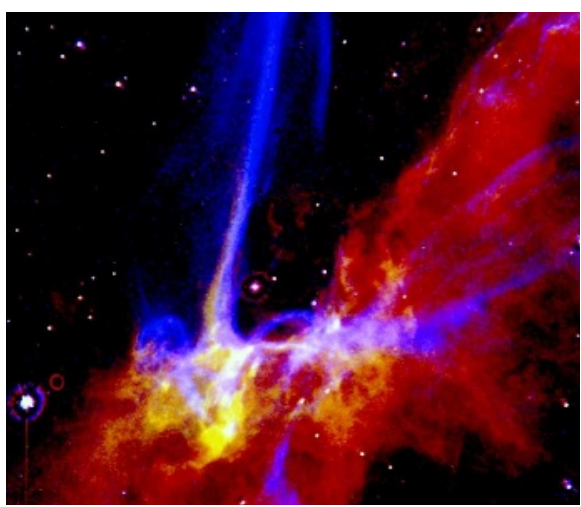

Supernovae offer clues to the expanding Universe.

decided by 3 October, according to NASA astrophysics division chief Jon Morse, who says that there were 50 applicants in total, some coming from all three teams. Neil Gehrels, principal investigator for the Swift Gamma Ray Burst Explorer at Goddard, will chair the group.

The decision took many by surprise. "I'm concerned," says Chuck Bennett, principal investigator for the ADEPT team at Johns Hopkins University in Baltimore, Maryland. "Three teams did a lot of work for a long time. I'm worried that hitting the reset button and starting again is going to set things back."

NASA has removed mention of the competition between the three projects from its websites; just weeks ago, it discussed deciding between the telescopes in 2009 .

It's not necessarily a bad move by the agencies, says Robert Cahn of Lawrence Berkeley National Laboratory in California, who was part of a task force convened in 2005 to examine the dark-energy question. The JDEM may have become too big and costly to have been managed well by the relatively small teams, but now NASA can adopt the best ideas from each, he says. "In some sense NASA seems to have made up its mind that it wants to do all three methods," he says. "It's certainly not working the way we expected but it might work out well."

Although the decision eliminates tension between the competing teams - all three presume they will share aspects of their once-secret designs for the science coordination group there is still tension between what the scientists want to do, and how much NASA and the DoE say they can afford. A 2007 National Academies report that endorsed the JDEM estimated that the three designs would cost more than US\$1.3 billion in total. But Morse has said that he can afford only a $\$ 600$-million mission, not including launch costs. The DoE has said it wants to pay about $25 \%$ of the overall costs. Eleven of the academy report's authors complained to NASA and the DoE in May that the science they envisioned the JDEM doing would not be possible at half the cost. "Wishful thinking does not engineer successful spacecraft," they wrote.

Eric Hand

\section{The telescope teams}

Three main teams designed space telescopes to measure how the Universe's expansion rate has changed over time, by performing surveys of objects from early in its history. Surveys require wide fields of view, and the need to look far back in time means that the telescopes would have to see infrared, the light with which the most distant and early objects glow. Both tasks are difficult from Earth. The teams emphasized different targets and techniques:

\section{Supernovae}

Dark energy was discovered in 1998 by using the assumption that all supernovae in a class shine with the same luminosity, which allows astronomers to calculate their distance from Earth more precisely. It was found

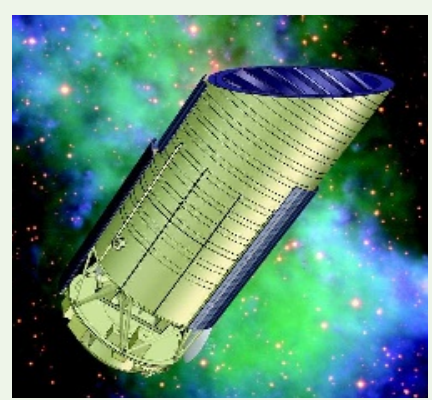

that more distant supernovae were receding more quickly than had been expected. The Supernova Acceleration Probe team initially emphasized this technique, although it later adopted all three methods.

\section{Weak gravitational lensing} A high-resolution picture of distant galaxies can reveal

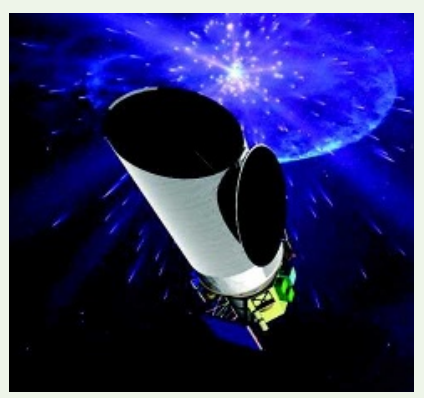

tiny distortions in their shapes caused by the 'lensing' of intervening dark matter. The uneven distribution of this matter, and how it changed with cosmological time, would be a proxy for how dark energy influenced the Universe's growth. The Dark Energy Space Telescope team emphasized a blend of lensing and supernovae.

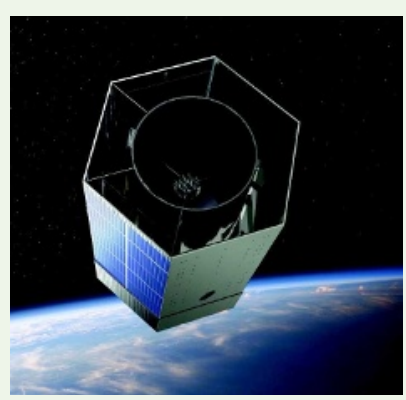

Baryonic acoustic oscillations Sound waves soon after the Big Bang created ripples in the distribution of galaxies. By comparing the ripples from galaxy clusters in the early Universe with ripples in later clusters, astronomers can deduce the effect of dark energy over time. The Advanced Dark Energy Physics Telescope team emphasized this approach. 